\title{
Three-dimensional flow in a barb field
}

\author{
B. Minor, E. Jamieson, C. D. Rennie \& R. D. Townsend \\ Department of Civil Engineering, University of Ottawa, Canada
}

\begin{abstract}
Barbs (or submerged groynes) are low-profile linear rock structures that are primarily used to prevent the erosion of stream banks. They are a variation of a groyne, similar to spur dikes and bendway weirs. Barbs are typically anchored, in series, to the outside bank in stream bends and extend in an upstream direction from the bank into the flow. This configuration redirects flow away from the outer stream bank and disrupts the velocity gradient close to the outer bank, encouraging sediment deposition adjacent to the barb. These interactions between the flow pattern and the sediment transport around the barb prevent erosion of the bank. Furthermore, vortices generated by the barb create local scour holes that can enhance aquatic habitat. A three-dimensional numerical model, Sediment Simulation in Intakes with Multiblock option (SSIIM), was used to examine the turbulent flow field and associated scour and depositional patterns due to a series of barbs in a mobile-bed channel bend. Previous physical model observations were used for model calibration and verification. Barb design was optimized to minimize scour. The parameters considered in the optimization were channel bend angle, barb alignment angle, and barb spacing. In this paper the spatial distribution of the three-dimensional flow field through a series of barbs in a $90^{\circ}$ channel bend are mapped and the impact on the flow field of subtle differences in barb geometry are examined. Special emphasis is placed on the influence of barb design on secondary currents in the meander bend and the generation of three-dimensional vortices, and the consequent impact on scour.
\end{abstract}

Keywords: channel bends, three-dimensional flow field, numerical models, mobile bed models, barbs, submerged groynes, scour, secondary flow, vorticity. 


\section{Introduction}

Historically, groyne structures have been built such that the groyne is exposed during moderate flows and only overtopped at the highest flows. More recently, there has been interest in low-profile submerged groynes such as barbs, because they require less material to construct and are less obtrusive. Barbs are typically oriented in the upstream direction and when anchored in series to the outside of a channel bend, are designed to redirect flow away from the outer stream bank and disrupt the velocity gradient and shear stress distribution close to the outer bank. In a channel bend, the highest rates of erosion are concentrated against the outer bank and just downstream of the bend apex. Barbs are designed to influence these forces by redirecting the flow from the outer bank and reducing maximum shear stress. As a result of this disruption, the location of the thalweg shifts away from the outside bank and a zone of subcritical flow is generated upstream of the barb structure along the outer bank. Therefore, when installed correctly, a series of barbs protects the outer bank from erosion and promotes sediment deposition. In addition, it has been observed that the local flow field and bed topography, including scour holes at barb tips, provide enhance aquatic habitat [1-3] and [4]. Design guidelines for barbs are currently based on field experience [5]. Better understanding of the interactions between the complex flow field and the sediment transport through the channel bend is required in order to predict the long-term performance of these structures.

Previous studies considered emergent groynes oriented perpendicular to the stream bank in a straight channel [6]. Recently, laboratory experiments using particle tracking velocimetry [7] and comprehensive three-dimensional numerical modelling [8] have provided detailed information regarding the flow pattern, shear layer and turbulence characteristics around a groyne head and between successive groynes. Changes in bed morphology due to the installation of a series of groynes were described [2]. Scour around a single submerged spur dike angled upstream in a straight channel has been studied [9]. More recently, the flow around a single submerged weir in a channel bend has been analyzed [10].

The scour patterns generated in a hydraulically narrow channel, associated with barbs installed at different spacings and angles relative to the bank in $90^{\circ}$ and $135^{\circ}$ channel bends, were examined [11]. The scour measurement data obtained from these experiments were used to calibrate and validate the numerical model presented in this paper. A three-dimensional numerical model was used to simulate the turbulent flow field and associated scour and deposition of bed sediments due to a series of barbs installed in both $90^{\circ}$ and $135^{\circ}$ channel bends. The impacts of various barb arrangements on the flow field and on the associated channel bed scour and deposition for the two bend geometries are presented elsewhere [12].

The present paper focuses on details of the three-dimensional flow field associated with various barb arrangements for the $90^{\circ}$ channel bend. The output from SSIIM includes the three-dimensional mean velocity and pressure fields. This mean flow field was utilized to calculate stream traces and the vorticity 
field using the commercial software Tecplot360. The stream traces are calculated possible particle trajectories, and provide effective flow visualization. The net result is a description of the vortex field around the barbs, which helps explain the observed patterns of scour and deposition.

\section{Experimental data}

The scour measurement data for flow in an open channel bend was collected in experiments performed in the Engineering Hydraulics Laboratory, University of Ottawa [11,13]. A laboratory channel, consisting of three rectangular-shaped detachable sections, was used to model the flow and associated scour patterns in a $90^{\circ}$ channel bend. The channel entrance section was $9.75 \mathrm{~m}$ long, followed by a $90^{\circ}$ bend section and a $2.44 \mathrm{~m}$ long exit channel. The entire channel had a constant width of $0.406 \mathrm{~m}$ and the upstream straight section had a constant slope of 0.001 . The entrance section was $0.254 \mathrm{~m}$ deep with a fixed bed. The bend and exit sections were $0.152 \mathrm{~m}$ deeper than the rest of the channel, allowing for a $0.152 \mathrm{~m}$ deep sand bed to be placed in these sections.

A series of experiments were run to measure the change in bed elevation in the channel for various barb arrangements installed along the outside bank of the bend section. These measurements were used to understand the effectiveness of each arrangement to promoting long term stability of the outside bank, which was assessed based on two criteria: (i) percent reduction achieved in scouring in the vicinity of the outside bank and (ii) degree to which the channel thalweg was moved from the outside bank towards the centre of the channel. The barb parameters that were varied for each experiment were barb alignment angle $(\theta)$, and group-type (A, B or C), which is defined by the location of the first barb in the group. The barb height at the bank was set at half of the mean flow depth, had a length $0.196 \mathrm{~m}$ and decreased with a $10^{\circ}$ slope away from the bank.

The sand in the bend and exit sections had a median diameter $0.85 \mathrm{~mm}$ with a geometric standard deviation of approximately 1.3. A constant discharge $0.0132 \mathrm{~m}^{3} / \mathrm{s}$ and average flow depth $0.102 \mathrm{~m}$ was maintained throughout the experiments. Further details of the experimental methods and data are presented elsewhere $[11,13]$.

\section{Numerical model}

The three-dimensional numerical model used in this study was Sediment Simulation in Intakes with Multiblock option (SSIIM), Version 1.1. This model, which was developed at the Norwegian University of Science and Technology [14], uses a three-dimensional structured and non-orthogonal grid to simulate flow and sediment transport. The three-dimensional Reynolds-averaged NavierStokes equations are solved to calculate water flow, with the k- $\varepsilon$ turbulence model to resolve the Reynolds stresses. The SIMPLE method is used to solve the pressure term. The suspended sediment transport is calculated with the convection-diffusion equation and the volumetric bedload transport is calculated using van Rijn's bedload transport formula. 


\subsection{Numerical simulation}

The three-dimensional numerical model was initially used to simulate the flow and sediment transport through the $90^{\circ}$ channel bend without barbs installed. The numerical model was then calibrated with the scour measurement data obtained from the experimental model results. The parameters varied to calibrate the numerical model to the experimental data were the bed roughness and time step. A Manning $\mathrm{n}=0.0167$ and a time step of 50 seconds yielded the most favourable results. A structured grid containing 123 cells in the longitudinal direction, 18 cells in the transverse (cross-stream) direction and 12 cells in the vertical direction was used to model the channel geometry in SSIIM. To ensure consistency with the experimental data, the channel geometry, discharge, flow depth, channel slope, sediment grain size and run time in the numerical model were all set equal to the values specified in the laboratory.

The results of the laboratory experiments found that for the $90^{\circ}$ channel bend, optimum bank protection was established for barb arrangement Group B with a barb alignment angle of $30^{\circ}$. Group B consisted of three barbs, with the first barb located at $1.41 \mathrm{~m}$ from the bend entrance.

The barb structures were modelled in SSIIM using a procedure described in [14], where cells defining the location of the barbs in the computational grid are blocked out. These cells are defined by fixed grid points with no slip conditions specified along the cell. The height (one half of the flow depth), length $(0.196 \mathrm{~m})$ and slope $\left(10^{\circ}\right.$ downward slope) remained consistent with the structures used in the laboratory experiments. However, due to limitations of the grid, the width of the barbs was wider than those used in the laboratory experiments.

\section{Results and discussion}

Six simulations were run for the $90^{\circ}$ channel, consisting of one reference run (no barbs installed) and five runs for five different barb arrangements. Predicted bed elevations in the numerical model reference run compared favourably to the measured bed elevations in the laboratory. The predicted patterns and magnitude of erosion and sediment deposition through the bend in the numerical model were similar to the measured data. A statistical comparison of the measured and predicted bed elevations for the $90^{\circ}$ reference channel gives a regression coefficient of determination $\left(\mathrm{r}^{2}\right)=0.8$. Details of the statistical analysis and a complete comparison between the measured and predicted change in bed levels are given in [12]. The principal objectives of this paper are: (i) to map the spatial distribution of the 3-D flow field through the barb groups and (ii) to show the predicted impact of subtle differences in barb geometry on the three-dimensional flow field.

It can be seen in Figure 1 that the maximum scour in the reference channel (no barbs) occurred along the outside of the bend, downstream of the bend apex. The predicted bed level changes for all five different barb arrangements are also shown. A comparison of these results shows how successful the placement of barbs along the outside of the bend was at shifting the thalweg towards the centre of the channel, away from the outside bank. The barbs have also contributed to 
reducing the total overall scour through the bend. However, while the impact of the barb structures on the magnitude and location of scour is most noticeable, the addition of barbs had little effect on sediment deposition along the inside of the channel bend. Initial bed elevation through the bend before a run was equal to $0.18975 \mathrm{~m}$. (In Figure 1, blue contours represent scour and orange/red represents aggradation). Figure 1 also shows that downstream of the bend apex, maximum scour occurs at the tip of the third barb for Group A and B and at the tip of the fourth barb for Group C.

Detailed measurements of the velocity field were not taken during the laboratory experiments. An average velocity of $0.29 \mathrm{~m} / \mathrm{s}$ was recorded. The average magnitude of the simulated depth-averaged horizontal velocity vectors was $0.23 \mathrm{~m} / \mathrm{s}$ and a maximum depth-averaged magnitude of $0.32 \mathrm{~m} / \mathrm{s}$ was observed $25^{\circ}$ into the channel bend in the centre of the channel.

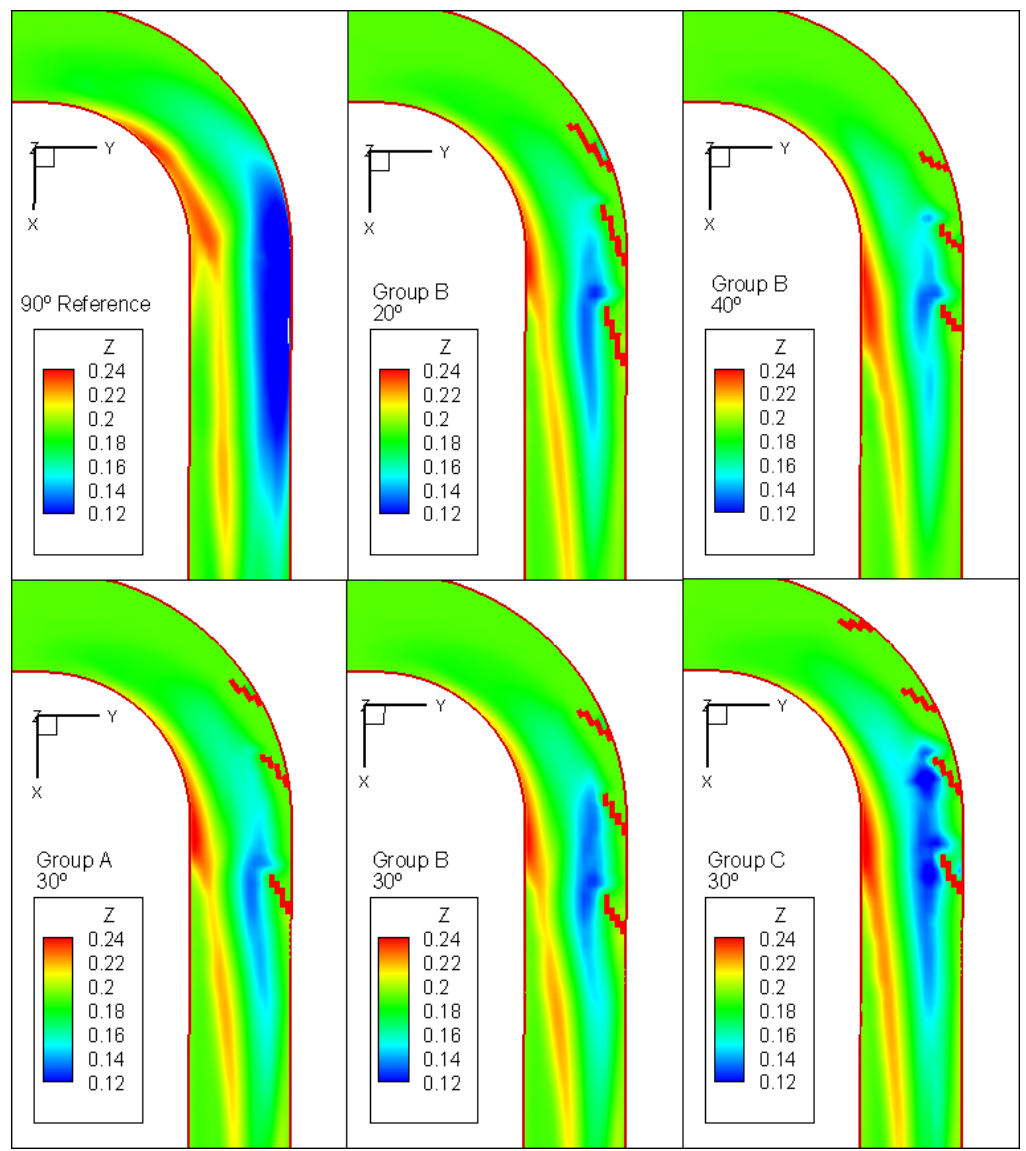

Figure 1: $\quad$ Predicted bed elevations $(Z)$ in metres for $90^{\circ}$ reference channel (top left) and five different barb arrangements. Flow is from top left to bottom right. 
The addition of barbs along the outer bank disrupts the typical structure of secondary flow present in a channel bend, where a single cell of rotation is described by the secondary velocity vectors that are directed downwards at the outer bank and upwards at the inner bank. With the addition of a barb, the velocity vectors at the outer bank become directed upwards and therefore, no longer attack the bank toe (Figure 2). At the tip of the barb, the velocity vectors are directed downwards, thereby contributing to the maximum scour at this location. The numerical results show that downstream of the barbs, two cells of secondary flow are formed, where the second cell is positioned immediately downstream of the structure and close to the outside bend (Figure 2). This second cell has a reversed orientation with flow at the bed now directed upwards and to the outside of the bend, resulting in lower velocities and subsequent sedimentation behind the barb.

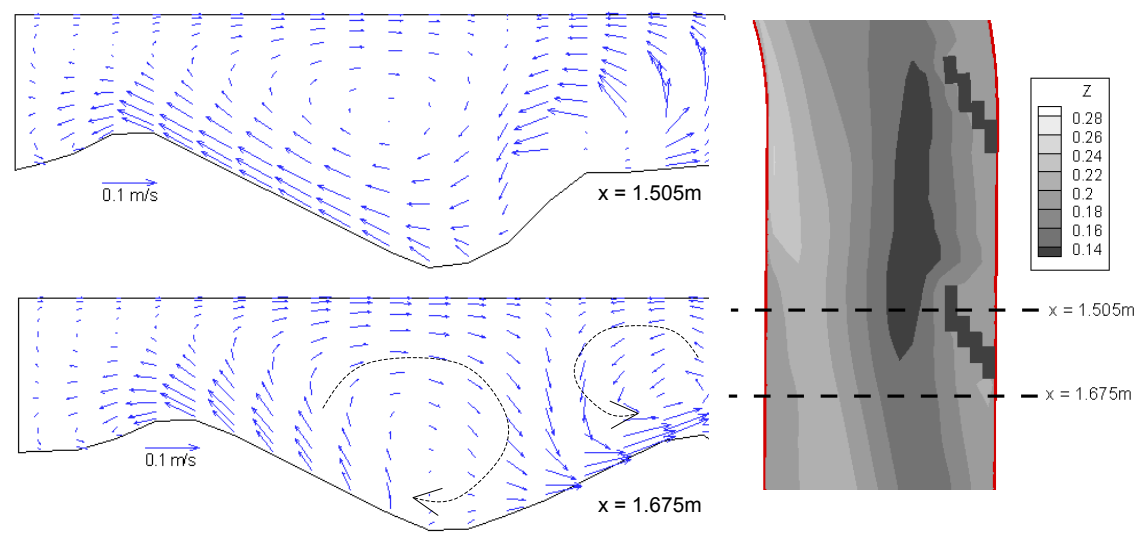

Figure 2: Cross-sectional velocity vectors at the tip of the third barb for Group-type B, $\theta=30^{\circ}$ (top) and just downstream (bottom).

The barbs are modelled as submerged structures, with their highest elevation nearest to the bank at one-half of the flow depth. This type of arrangement means that the structures are continuously overtopped, resulting in plunging flow as it passes over the structure (Figure 3). Unlike typical groyne structures, which are traditionally emergent (i.e. no regular overtopping) and perpendicular to the flow, the separation of the flow is less significant for barbs because they are submerged and orientated in a more streamlined direction.

If a structure (object) obstructs a high Reynolds number flow, due to the significant velocity gradient created and corresponding low pressure, the boundary layer developed against the object will separate from the wall. The separated flow will generate a downstream shear layer with the main flow and vertical vortical structures may develop. Conversely, the plunging of the flow causes regions of high velocity above the barbs and low velocity between the barbs. This velocity gradient can induce a cross-stream (transverse) vortex. However, in this model, cross-stream vortical structures were not directly observed with the stream traces. This is likely for two reasons: (1) the barb 
heights relative to depth were too small to generate a velocity gradient (or flow separation) sufficient to produce observable vortices and/or (2) the resolution of the computational grid in the area of the barbs may not have been adequate for resolving such details of the flow.

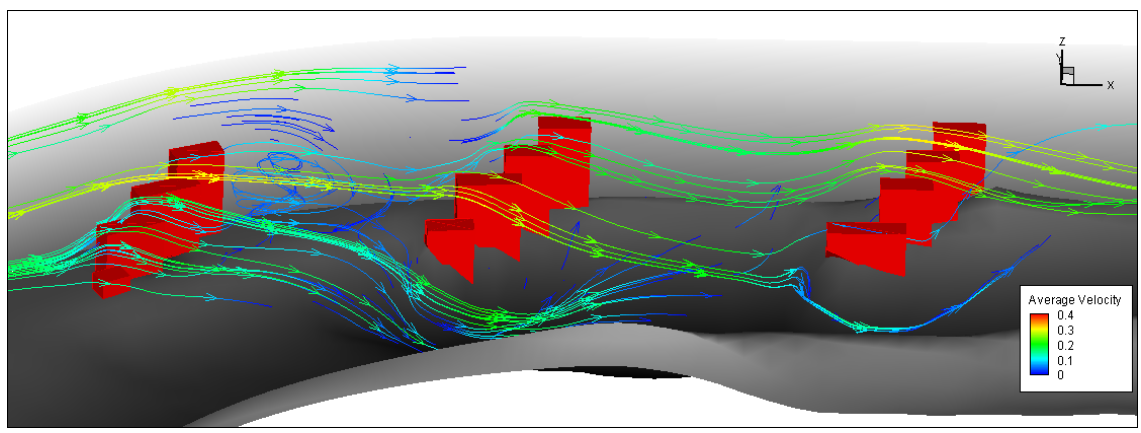

Figure 3: Plunging action of flow over the barbs illustrated by calculated stream traces, coloured according to average velocity $(\mathrm{m} / \mathrm{s})$.

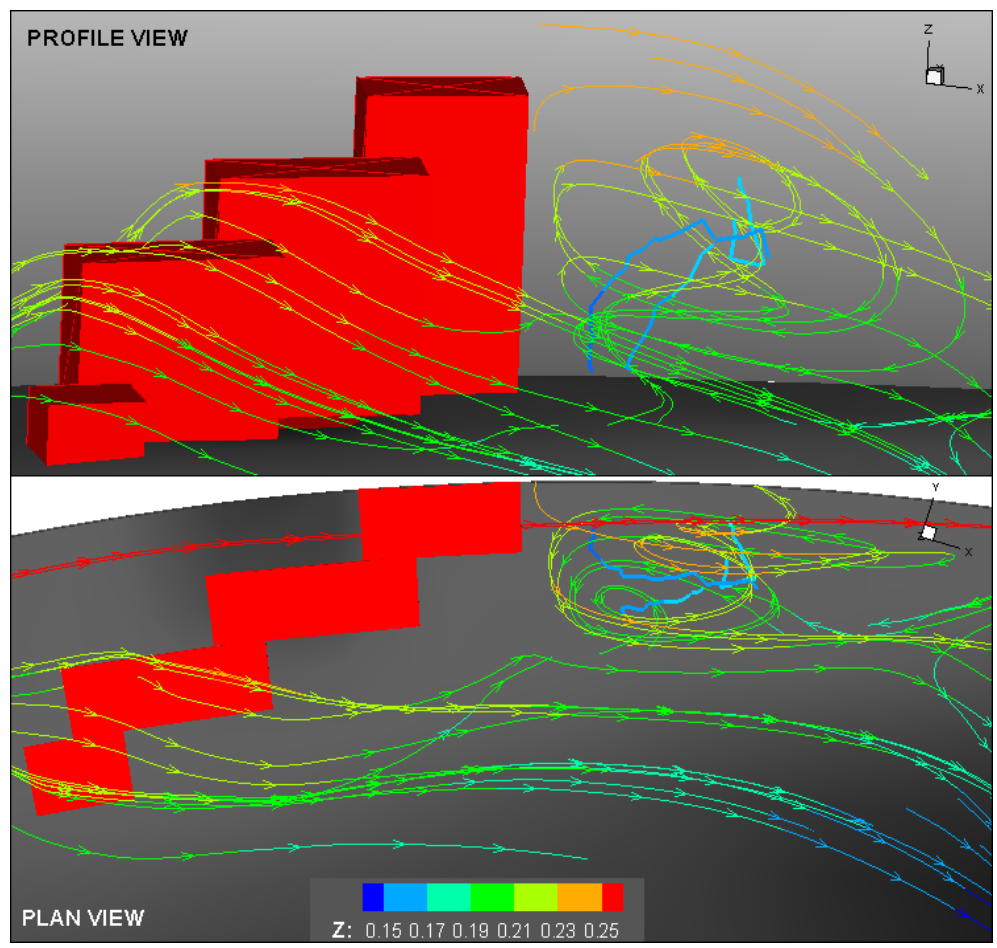

Figure 4: $\quad$ Predicted streamlines (shown in colour according to elevation, $\mathrm{Z}$ $(\mathrm{m})$ ) and vortex core (blue) for the first barb in Group B, $\theta=30^{\circ}$ arrangement. 
A detailed view of the stream traces for the first barb in Group B $\left(\theta=30^{\circ}\right)$ shows a region of recirculation and the presence of a vertically oriented vortex behind the first barb (Figure 3 and 4). In this case, the flow is rotating in a clockwise direction as it rises. In plan view (Figure 4, bottom), the vortex is positioned behind the tallest section of the barb, where horizontal flow separation would be most significant due to the largest velocity gradient between the flow in the main channel and the low velocities behind the structure.

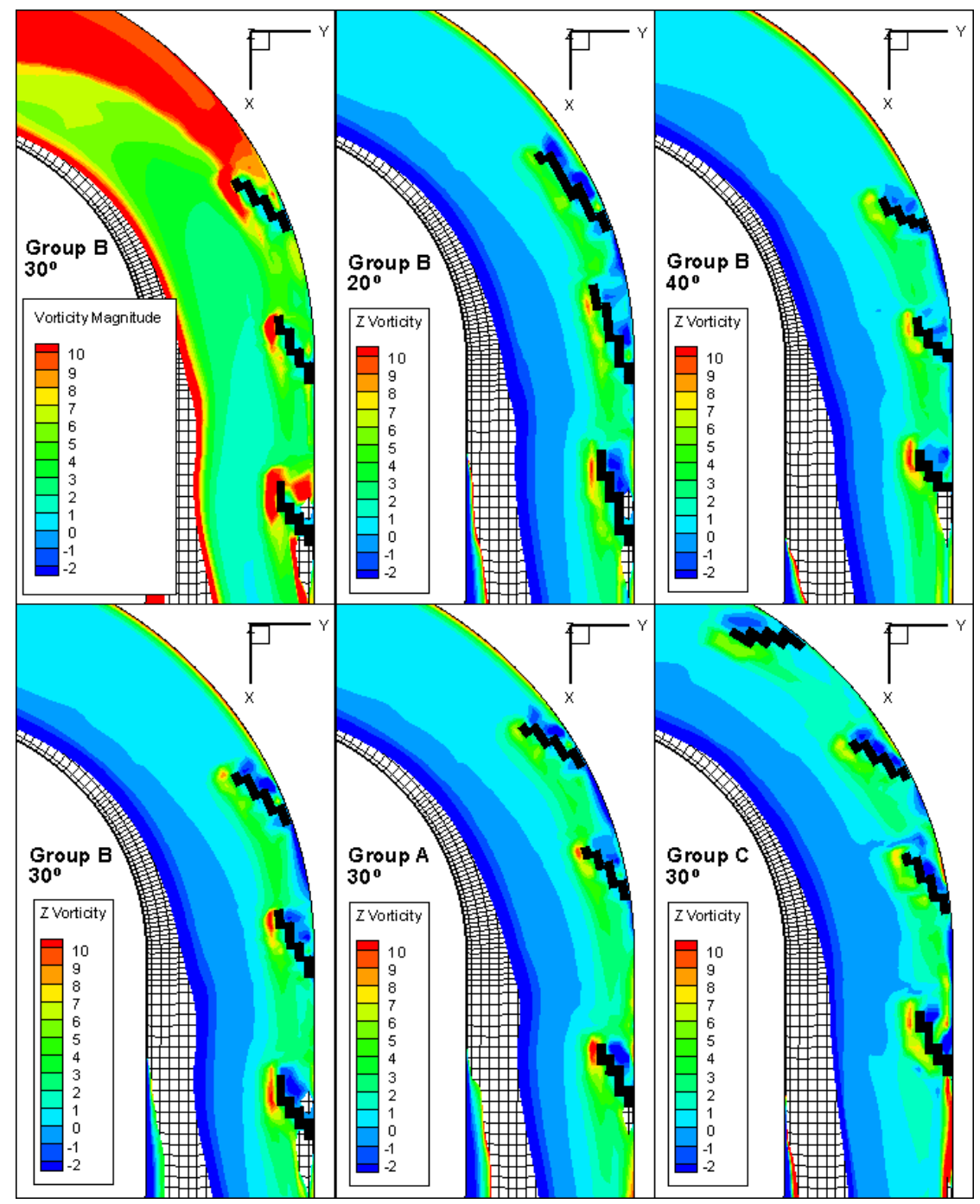

Figure 5: Calculated vorticity magnitude $\left(\mathrm{s}^{-1}\right)$ for Group B $\left(\theta=30^{\circ}\right)$ (top left) and Z-vorticity $\left(\mathrm{s}^{-1}\right)$ for all barb arrangements. (Contours of vorticity are at $\mathrm{Z}=0.1950 \mathrm{~m}$ and flow is from top left to bottom right). 
The magnitude of vorticity through the barb field was calculated and results are shown in Figure 5. Regions of high vorticity are present at the tip of all three barbs for Group B $\left(\theta=30^{\circ}\right)$ (Figure 5, top left). Vorticity magnitudes were calculated to be highest at the tip of all barbs relative to the magnitude of vorticity throughout the barb field. Further, vertical (or Z) vorticity, which can contribute to local scour of the bed, is similarly high at the barb tips and low immediately upstream of the barbs for all Group-types and barb angles (Figure 5). This suggests that vortices with vertical axes generated from flow separation at the barb tip dominate the vorticity field.

In all five different barb arrangements, the location of maximum scour occurred at the tip of the last barb in the downstream direction, while no scour was measured at the tip of the first (or in some cases second) barb (Figure 1). This pattern corresponds to the results of the calculated Z-vorticity, where maximum $Z$-vorticity close to the bed $(Z=0.1950 \mathrm{~m})$ is at the tip of the second last and last barb in the downstream direction (Figure 5). As a result, a detailed description of the vertical $(Z)$ vorticity field has helped to explain the observed patterns of scour around the barb tips. Further, by comparing the patterns and magnitude of scour and deposition around the barb (Figure 1) with detailed contours of vertical vorticity (Figure 5), it is possible to estimate the relative magnitude of vorticity required to generate a scour hole or initiate deposition. The observed relationship between local scour and vorticity will help better understand and predict the changes in bed level for different flow fields associated with different barb geometries and arrangements in series.

\section{Conclusions}

Three-dimensional numerical modelling results have provided detailed inspection of the predicted three-dimensional velocity field in the vicinity of barbs. Further analysis, including the generation of stream traces and the calculation of vorticity, has provided a comprehensive view of the threedimensional characteristics of flow in a barb field. The pattern and magnitude of erosion and deposition of sediment in a channel is dependent on the characteristics of the flow field. As flow in a channel bend is disrupted by the addition of barbs (or other in-stream structures) this pattern will change. Understanding the flow in detail provides a better means of predicting the resulting bed level changes for various barb geometries and orientations, resulting in better optimization of barb design for stream bank protection or other requirements.

\section{References}

[1] Shields, F.D., Knight, S.S. \& Cooper, C. M., Addition of spurs to stone toe protection for warmwater fish habitat rehabilitation. J. American Water Res. Ass., 34, pp. 1427-1436, 1998.

[2] Sukhodolov, A., Uijttewaal, W.S.J. \& Engelhard, C., On the correspondence between morphological and hydrodynamic patterns in 
groyne fields. Earth Surface Processes and Landforms, 27, pp. 289-305, 2002.

[3] Engelhardtm C., Kruger, A., Sukhodolov A., A study of phytoplankton spatial distributions, flow structure and characteristics of mixing in a river reach with groynes. Journal of Plankton Research, 26(11), pp. 1351-1366, 2004.

[4] Schwartz, R. \& Kozerski, H-P., Entry and deposits of suspended particulate matter in groyne fields of the middle Elbe and its ecological relevance. Acta Hydrochimica et Hydrobiologica, 31(4-5), pp. 391-399, 2003.

[5] Technical Note 23: Design of Stream Barbs, U.S. Department of Agriculture (USDA), Natural Resources Conservation Service, Portland Oregon, 2005.

[6] Uijttewaal, W.S.J., Lehmann, D. \& van Mazijk, A., Exchange process between a river and its groyne fields: Model experiments. J. Hydraulic Research, 127(11), pp. 928-936, 2001.

[7] Uijttewaal, W.S.J., Effects of groyne layout on the flow in groyne fields: Laboratory experiments. J. Hydraulic Eng., 131(9), pp. 782-791, 2005.

[8] McCoy, A., Constantinescu, S.G. \& Weber, L., Coherent structures in a channel with groyne fields: A numerical investigation using LES. Presented at the World Water and Environmental Resources Congress, Alaska, May 2005.

[9] Kuhnle, R.A., Alonso, C.V. \& Shields, F.D. Jr., Local scour associated with angled spur dikes. J. Hydraulic Eng., 128(12), pp. 1087-1093, 2002.

[10] Jia, Y., Scott, S., Xu, Y., Huang, S. \& Wang, S.S.Y., Three-dimensional numerical simulation and analysis of flows around a submerged weir in a channel bendway. J. Hydraulic Eng., 131(8), pp. 682-693, 2005.

[11] Matsuura, T. \& Townsend, R., Stream-barb installations for narrow channel bends - a laboratory study. Canadian Journal of Civil Engineering, 31(3), pp. 478-486, 2004.

[12] Minor, B., Rennie, C.D. and Townsend, D.R., Barbs (submerged groynes) for river bend bank protection: Application of a three-dimensional numerical model. Canadian Journal of Civil Engineering, in review.

[13] Matsurra, T., Stream-Bank Protection in Narrow Channel Bends Using 'Barbs': A Laboratory Study. M.A.Sc Thesis, Department of Civil Engineering, University of Ottawa, Canada, 2004.

[14] Olsen, N.R.B., A Three-dimensional Numerical Model for Simulation of Sediment Movements in Water Intakes with Multiblock Option: User's Manual. Department of Hydraulic and Environmental Engineering, Norwegian University of Science and Technology, Trondheim, Norway, 2004. 